\title{
A rheostat for immune responses: the unique properties of PD-1 and their advantages for clinical application.
}

\section{$\operatorname{AUTHOR}(\mathrm{S})$ :}

Okazaki, Taku; Chikuma, Shunsuke; Iwai, Yoshiko; Fagarasan, Sidonia; Honjo, Tasuku

\section{CITATION:}

Okazaki, Taku ... [et al]. A rheostat for immune responses: the unique properties of PD-1 and their advantages for clinical application.. Nature immunology 2013, 14(12): 1212-1218

\section{ISSUE DATE:}

2013-12

URL:

http://hdl.handle.net/2433/193052

\section{RIGHT:}

(c) 2013 Nature America, Inc.; This is not the published version. Please cite only the published version.; この論文は出版社版でありません。引 用の際には出版社版をご確認ご利用ください。 


\section{A rheostat for immune responses- the unique properties of PD-1 and their advantage for clinical application}

Taku Okazaki ${ }^{1, \dagger}$, Shunsuke Chikuma ${ }^{2, \dagger}$, Yoshiko Iwai ${ }^{3}$, Sidonia Fagarasan ${ }^{4}$, and Tasuku Honjo $^{2 *}$

${ }^{1}$ Division of Immune Regulation, Institute for Genome Research, University of Tokushima, 3-18-15 Kuramoto, Tokushima, 770-8503 Japan

${ }^{2}$ Department of Immunology and Genomic Medicine, Graduate School of Medicine, Kyoto University, Yoshida-Konoe Sakyo-ku, Kyoto, 606-8501 Japan

3 Department of Molecular Biology, School of Medicine, University of Occupational and Environmental Health, Iseigaoka, Yahatanishi-ku, Kitakyushu 807-8555, JAPAN ${ }^{4}$ Laboratoryfor Mucosal Immunity, Center for Integrative Medical Sciences IMS-RCAI, RIKEN Yokohama Institute, 1-7-22 Suehiro-cho, Tsurumi, Yokohama, Kanagawa, 230-0045, Japan.

$\dagger$ These authors contributed equally to this work.

*Corresponding author: Dr. Tasuku Honjo, Yoshida-Konoe, Sakyo-ku, Kyoto, 606-8501, JAPAN

Tel: +81-75-753-4371 Fax: +81-75-753-4388 E-mail: honjo@mfour.med.kyoto-u.ac.jp 


\begin{abstract}
PD-1, a negative coreceptor expressed on antigen-stimulated T and B cells, appears to serve as a rheostat of the immune response. The molecular mechanisms of PD-1 functions in conjunction with the mild, chronic and strain-specific autoimmune phenotypes observed in PD-1-deficient mice, as opposed to the devastating fatal autoimmune disease observed in CTLA-4-deficient mice, suggest that immune regulation by PD-1 is rather antigen-specific and primarily cell-intrinsic. These unique properties make PD-1 a powerful target for immune therapy, with highly effective clinical applications for cancer treatment.
\end{abstract}


Antigen receptors are known to have a broad specificity with a wide range of affinity. This intrinsic characteristic of antigen receptors inevitably makes thymic negative selection incomplete for avoiding self-reactive immune responses in the periphery. It is therefore essential that the antigen recognition signaling system is equipped with a rheostat, which regulates the threshold of antigen response for balanced immune-physiology. Deficiency of PD-1 (Pdcd1, CD279), a negative coreceptor isolated by cDNA subtraction with unknown function in $1992^{1}$, causes the development of different autoimmune phenotypes on various genetic backgrounds of mice ${ }^{2-7}$ PD-1 was thus shown to be a critical negative co-receptor regulating the threshold of antigen response of $\mathrm{T}$ and $\mathrm{B}$ cells in the periphery. $\mathrm{PD}-1$ activation upon interaction with its ligands (PD-Ls) ${ }^{8,9}$, dephosphorylates key proteins immediately downstream of the antigen receptor ${ }^{10-14}$, which endows PD-1 with immunoregulatory functions.

By regulating the function of $\mathrm{CD} 8^{+} \mathrm{T}$ cells, $\mathrm{PD}-1$ modulates immunity against infections. PD-1 deficiency renders the mice resistant to viral infection, by reducing the antigen recognition threshold and increasing the cytotoxic lymphocyte activity of CD8 $8^{+}$ $\mathrm{T}$ cells. The regulatory function of PD- 1 on the threshold of $\mathrm{T}$ cell immune responses is sustained by the observation that PD-1 deficiency induces suppression of tumor growth and tumor metastasis in mice ${ }^{15,16}$. Furthermore, PD-1 is essential for generation and selection of high quality, high affinity antibodies by regulating the properties and number of antigen-stimulated $\mathrm{CD} 4^{+} \mathrm{T}$ cells ${ }^{17,18}$. This regulation is critical not only for antibody-mediated memory but also for control of the gut microbiota by the adaptive immune system. This latter aspect opens a new perspective of PD-1 involvement in the 
fine-tuned regulation of symbiotic relationships between the immune system and the gut microbiota and in regulation of other physiological systems of the body via interaction with the whole microbial products.

PD-1 has unique properties compared with CTLA-4, the other major negative coreceptor expressed on T cells. CTLA-4 has both cell-intrinsic (CTLA-4 on effector cells) and cell-extrinsic (CTLA-4 on Foxp3 ${ }^{+} \mathrm{T}$ cells) activities, which make the autoimmune phenotypes of CTLA-4 deficiency very severe and antigen non-specific ${ }^{19}$, 20. In contrast, the effects of PD-1 engagement are primarily cell-intrinsic. This cell-intrinsic function of PD-1, as well as the regulation of PD-1 expression is probably responsible for the relatively milder and more chronic symptoms of PD-1 blockade by either antibody or genetic manipulation. These subtle effects of PD-1 blockade are currently exploited in translational medicine for boosting the immune responses in several pathologies.

In this Review, we discuss the unique properties of PD-1 as a rheostat of immune regulation. We focus on the characteristics that make PD-1 distinct from other negative regulators (co-inhibitory receptors, transcription factors or cytokines), and discuss the immune mechanisms that endow PD-1 with unique modulatory functions critical for immunological therapy against tumors. We present an overview of the PD-1 research in the course of the past two decades, and highlight how the discovery of PD-1 led to appreciation of the delicate regulation of antigen stimulation for immunophysiology and its exciting application in tumor treatment. Currently, PD-1 offers one of the best examples of scientific translation from bench to bedside and a 
powerful demonstration to us all- scientists, pharmaceutical companies and funding agencies, of the extreme importance of basic research for progress in medicine.

\section{PD-1 regulates the threshold of immune response}

The activation of lymphocytes depends primarily on the antigen recognition through antigen specific receptors, whereas additional inputs through co-receptors fine-tune this activation signal to regulate its strength, duration and property. The fate of lymphocytes after antigen encounter is determined by the integration of the stimulatory and inhibitory signals from co-receptors, each of which has its unique characteristics. CD28 and CTLA-4, which provide positive and negative co-stimulation, respectively, upon interaction with either of two shared ligands, B7.1 (CD80) and B7.2 (CD86), are the prototypes of such co-receptors. PD-1, together with several other proteins like inducible costimulator (ICOS, CD278) and B and T cell attenuator (BTLA, CD272) belongs to the CD28 co-receptor family. Like CD28 and CTLA-4, PD-1 has two ligands, PD-L1 (Pdcd1lg1, B7-H1, CD274) ${ }^{8}$ and PD-L2 (Pdcd1lg2, B7-DC, CD273) ${ }^{9}$. PD-1 lacks the membrane proximal cysteine residue required for homodimerization which is characteristic to other CD28 family members. As such, PD-1 exist as a monomer on the cell surface ${ }^{21-23}$. Due to its interaction with the adaptor protein complex AP2, CTLA-4 is subject to continuous clathrin-dependent endocytosis and as such is barely detected on the cell surface ${ }^{24}$. In contrast, PD-1 lacks an AP2 binding motif, which may allow its sustained expression on the cell surface of activated $\mathrm{T}$ cells. 
Ligand engagement of PD-1 during antigen recognition induces cross-linkage of the antigen receptor complex with PD-1. This causes phosphorylation of the tyrosine residue in the immunoreceptor tyrosine-based switch motif (ITSM; TxYxxL/I) of PD-1 and recruits the protein tyrosine phosphatase SHP-2, which dephosphorylates and inactivates proximal effector molecules such as Syk in B cells and ZAP70 in T cells ${ }^{10-14}$. The immediate outcome of PD-1 stimulation is the inhibition of cell growth and cytokine secretion (Fig. 1).

PD-1 is expressed on double negative $\alpha \beta$ and $\gamma \delta \mathrm{T}$ cells in thymus and on activated T, B, NK, NKT and myeloid cells in the periphery ${ }^{25-29}$. Activation-induced expression of PD-1 suggests that PD-1-dependent inhibition functions in later phases of the immune response (e.g. sustained activation, secondary response, effector phases etc. $)^{25}$. When naive DO11.10 T cells from PD-1-deficient and-sufficient transgenic mice were stimulated with $\mathrm{PD}-\mathrm{L1}^{+}$antigen presenting cells (APCs) in vitro, PD-1 deficiency increased the number of cells with more than 3 divisions, but not those with 1 to 2 divisions, suggesting that PD-1 expression is induced during the first to second round of division, after which $\mathrm{T}$ cells became sensitive to PD-L1 on APCs ${ }^{30}$. PD-1 begins to function after $\mathrm{T}$ cells recognize their cognate antigen and start the activation process. The amount and the source of antigen determine the strength and kinetics of $\mathrm{T}$ cell activation, and thus the amount and kinetics of PD-1 expression. In addition, the expression of PD-Ls also varies depending on the cell type and their activation status. Therefore, PD-1-dependent inhibition is very sensitive to the context, and thus the 
antigen, which may explain why PD-1 deficiency apparently augments antigen-specific immune responses, although neither PD-1 nor PD-Ls are antigen specific.

Anatomical variation of PD-Ls expression critically affects the target specificity. PD-L1 is highly expressed on non-lymphoid cells, including parenchymal cells, tumor cells and virus-infected cells, which allows PD-1 to directly inhibit effector functions against target cells (Fig. 2). In an in vitro experiment, PD-L1-expressing tumor cells showed relative resistance to cytotoxicity, suggesting that the engagement of PD-1 on $\mathrm{CD}^{+} \mathrm{T}$ cells by PD-L1 expressed on specific target cells results in the inhibition of T cell-mediated cytotoxic activity ${ }^{15}$. In autoimmunity, PD-L1 is induced on tissue-parenchymal cells in the affected organs 31,4 . In experiments using bone-marrow chimera and adoptive transfers, PD-L1 expression on parenchymal cells rather than hematopoietic cells was shown to protect against autoimmune diabetes ${ }^{28}$. In contrast to PD-L1 expressed in many tissues, the expression of PD-L2 is restricted to professional APCs (DCs, B cells etc.) ${ }^{27}$. PD-L2 might be involved in the different aspects of immune regulation, such as T-B collaboration for production of appropriate antibodies (discussed below)

Although most experimental data support the idea that PD-1 inhibits antigen stimulation in a cell-intrinsic manner upon interaction with either of its two ligands, there are several observations that could be explained by alternative mechanisms, including reverse signaling through PD-Ls (reviewed in ${ }^{32}$ ). However, the physiological contributions of these alternative pathways are currently uncertain, partly because no clear molecular mechanisms have been identified. 


\section{PD-1 regulates peripheral tolerance}

PD-1 deficiency causes the loss of peripheral tolerance and the subsequent development of autoimmunity in mice ${ }^{2,3}$. Aged $P d c d 1^{-/}$mice develop lupus-like glomerulonephritis and arthritis on a C57BL/6 background, while they develop dilated cardiomyopathy (DCM) by generation of anti-troponin I antibodies on the BALB/c background ${ }^{3,33}$. This observation was the first clear experimental demonstration of autoimmune basis of DCM, and provided the rationale for immuno-adsorption therapy to this deadly disease $^{34}$. The tissue-damaging autoantibodies produced in BALB/c- $P d c d l^{-/}$mice require class switching and/or somatic hypermutation, because DCM and gastritis development in these mice is dependent on a cytidine deaminase AID, a master regulator of class switching and somatic hypermutation ${ }^{30}$. As discussed below, the self-reactive antibodies in PD-1-deficient mice are likely generated in germinal centers (GC) induced systemically by dysregulated gut microbiota, and aided by GC T cells with pro-inflammatory properties (i.e. producing more IFN- $\gamma$ but less IL-21) ${ }^{18}$.

In addition, backcrossing of $P d c d 1^{-/}$mice on various other backgrounds revealed that PD-1 deficiency induces autoimmunity in diverse target organs, depending on the genetic background of the mice $e^{4-7}$. The variations in the disease phenotype depending on the genetic background suggest that absence of PD-1 may exaggerate strain-specific autoimmune susceptibilities. This indicates that PD-1 regulation is apparently (as in not absolutely) antigen-specific. The target specificity of PD-1-dependent regulation of autoimmunity also holds true for the association between 
single nucleotide polymorphisms in the PD-1 gene with various kinds of human autoimmune diseases including systemic lupus erythematosus, type I diabetes, multiple sclerosis, rheumatoid arthritis, Grave's disease and ankylosing spondylitis ${ }^{35-37}$. Some of these diseases associates with relatively novel effector helper $\mathrm{T}$ cell population producing IL-17 (Th17). In experimental autoimmune encephalitis, a model for human multiple sclerosis, PD-1 obviously down-regulated IL-6, a pro-inflammatory cytokine from innate immune cells, required for Th17 differentiation ${ }^{38}$. Possibly PD-1 regulates both innate and lymphocyte responses to maintain self-tolerance.

PD-1 was shown to collaborate with another inhibitory co-receptor, LAG-3 in the regulation of autoimmunity ${ }^{30}$. Mice deficient for both LAG-3 and PD-1 on the BALB/c background died of autoimmune myocarditis by 5 weeks of age. In vitro experiments revealed that PD-1 and LAG-3 synergistically inhibit the antigen-induced activation of T lymphocytes. Although LAG-3 was proposed to suppress the activation of $\mathrm{CD}^{+} \mathrm{T}$ cells by competing with $\mathrm{CD} 4$ for MHC class II binding, the precise molecular mechanism and its biological function remains largely unknown ${ }^{39,40}$.

\section{Distinct physiological functions of PD-1 and CTLA-4}

As already mentioned, the autoimmune phenotypes of $P d c d 1^{-/}$mice are generally much milder, confined to specific organs and have a rather late onset compared with $\mathrm{Ctla4}^{-/}$ mice. In the latter, $\mathrm{T}$ cells that are non-specifically activated invade various organs, resulting in premature death with GVH-like disease, irrespective of the genetic background $^{20}$. This drastic phenotype is reminiscent of Foxp3 and TGF- $\beta 1$-deficient 
mice ${ }^{41,42}{ }^{43}$. In fact, $>90 \%$ of CTLA-4 expressing cells also express FoxP3 ${ }^{20}$. Interestingly, the severe phenotypes seen in mice deficient for CTLA-4, TGF- $\beta 1$ or Foxp3 appear to be rather caused by cell-extrinsic mechanisms, based on the following observations: co-transfer of CTLA-4-deficient bone marrow cells along with wild-type bone marrow cells could rescue the autoimmune phenotype caused by CTLA-4-deficient bone marrow cells alone ${ }^{44}$; adoptive transfer of bone marrow cells from wild-type mice can rescue the lethal phenotypes of Foxp3-deficient scurfy mice ${ }^{45}$; and TGF- $\beta 1$ inhibits inflammatory cells and promotes the development and function of Foxp $^{+} \mathrm{T}$ cells ${ }^{46}$. In contrast, the autoimmune manifestations in $P d c d 1^{-/-}$mice could not be corrected by co-transfer with wild-type $\mathrm{T}$ cells, suggesting that the phenotype of PD-1-deficient mice are primarily attributable to cell-intrinsic mechanisms ${ }^{5,30}$ (Fig. 3).

A large number of reports suggest that CTLA-4 plays critical roles in regulation of immune responses mainly by modulating the function of Foxp $3^{+} \mathrm{T}$ cells (reviewed in ${ }^{20}$ ). However, several groups reported that CTLA-4-deficient Foxp3 ${ }^{+} \mathrm{T}$ cells retain their suppressive functions, suggesting that multiple mechanisms may operate under different assay conditions. Similarly, conflicting data were reported regarding whether Foxp $3^{+} \mathrm{T}$ cells from TGF- $\beta 1$-deficient mice were able to suppress the autoimmune phenotypes ${ }^{47}$. It will be critical to clarify whether these and other inhibitory molecules function in parallel, in series or both for the negative regulation of immune responses.

Although Foxp $3^{+}$T cells express PD-1, the contribution of PD-1 to their suppressive function seems to be small, if any, at least in an in vitro suppression assay ${ }^{6}$. In fact, the 
Foxp $^{+} \mathrm{T}$ cell compartment is larger in PD-1 deficient mice (SF, unpublished data). Since PD-1 deficient T cells are prone to be activated and Foxp3 expression is induced in activated $\mathrm{T}$ cells ${ }^{48}$, it is reasonable that PD-1-deficient mice have increased frequencies of Foxp $3^{+} \mathrm{T}$ cells.

The inhibitory mechanisms of PD- 1 and CTLA- 4 are quite distinct, because CTLA-4 completely blocks CD28 co-stimulation through its stronger affinity to B7s ${ }^{49,50}$, while the inhibitory effect of PD-1 on TCR signaling and CD28 co-stimulation is indirect and thus less complete and slower. The inhibitory function of PD-1 mostly depends on its recruitment of SHP-2, while CTLA-4 signaling involves a wider variety of molecules including SHP-2, the phosphatase PP2A and AP2 ${ }^{12}$. The AP2-dependent endocytosis of CTLA-4 together with the B7 ligands has been suggested to be central in the regulatory function of Foxp3 $3^{+}$cells ${ }^{51}$. Although it remains to be seen whether this represents the main mechanism for the cell-extrinsic effects of CTLA-4, it is likely that CTLA-4 has both cell-intrinsic and cell-extrinsic effects and functions as an 'on-and-off ', rather than a 'rheostat', negative regulator. As such, the inhibitory functions of PD-1 appear to be quite distinct from that of other negative regulators, with PD-1 deficiency affecting antigen specific autoimmune responses, whereas deficiency in other negative regulators showing more systemic, antigen non-specific phenotypes.

\section{Regulation of antibody responses by PD-1}

Humoral immune responses are generated by $\mathrm{T}$ cell-independent and $\mathrm{T}$ cell-dependent pathways. Interestingly, both types of immune responses are controlled by PD-1. PD-1 
is highly expressed by innate type B cells, like peritoneal cavity B1 cells, after their activation by antigens ${ }^{52,53}$ and appears to suppress the B cell receptor (BCR)-induced expansion of B1 cells and their differentiation into long-lived IgG plasma cells ${ }^{54}$. Thus, the engagement of PD-1 on B1 cells by its ligands (likely expressed by macrophages located in the red pulp of the spleen, where B1-derived plasmablasts preferentially locate, and by the plasma cells themselves) contributes to down modulation of innate-like responses and facilitates the "take over" by the adaptive responses through longer-lived plasma cells and memory cells generated in the $\mathrm{GC}^{55}$.

The GC are specialized microenvironments, where antigen-activated B cells interacting with $\mathrm{T}$ cells upregulate AID and undergo the two genetic alterations required for effective and long lasting immune responses, namely class switch recombination and somatic hypermutation ${ }^{56}$. PD-1 appears to regulate these two GC-dependent immunological functions. PD-1 is highly expressed on the two subsets of CD4 ${ }^{+} \mathrm{T}$ cells present in the GCs: $\mathrm{T}$ follicular helper $\left(\mathrm{T}_{\mathrm{FH}}\right)$ cells - defined as $\mathrm{CXCR}^{+}$PD- ${ }^{\text {hi }}$ Foxp3cells ${ }^{57}$, and $\mathrm{T}$ follicular regulatory $\left(\mathrm{T}_{\mathrm{FR}}\right)$ cells - defined as $\mathrm{CXCR}^{+}$PD-1 ${ }^{\text {hi }}$ Foxp3 $^{+}$ cells ${ }^{58-60}$. Some GC B cells also express PD-1 ${ }^{17}$, while PD-L1 and PD-L2 are expressed within the GCs, with the B cells in the light zone of the GCs and memory B cells highly expressing PD-L2 ${ }^{17,18}$. Thus, PD-1 on GC $\mathrm{T}$ cells is mostly engaged by PD-L2 expressed on GC B cells, and this interaction probably modulates the GC reaction in situ. Indeed, the B cell-intrinsic expression of PD-L2 was shown to be required for optimal generation of antibody-secreting cells ${ }^{17}$. However, the phenotypes of $P d c d 1 \lg 1^{-/-} P d c d 1 \lg 2^{-/-}$double deficient mice and $P d c d 1^{-/-}$mice were more pronounced 
than those of the $P d c d l \lg 2^{-/-}$mice, at least after systemic immunization. Thus, constitutive PD-L1 expression on B cells also probably contributes to PD-1 signaling and affects systemic GC responses. Lack of ligand interactions for PD-1 causes inappropriate immune responses, manifested by reduced formation of long-lived IgG plasma cells in the bone morrow ${ }^{17}$ and deficient qualities of IgA plasma cells in the gut 18

Mechanistically, PD-1-deficiency in mice leads to expansion of $\mathrm{T}_{\mathrm{FH}}$ cells with reduced capabilities of IL-21 production ${ }^{17,18}$. IL-21 is important for GC formation and function and its absence affects $\mathrm{B}$ cell proliferation and differentiation into memory B cells and plasma cells ${ }^{61-63}$. PD-1 deficiency causes enhanced production of other cytokines by $\mathrm{CD}^{+} \mathrm{T}$ cells located outside GCs (i.e Pre-GC $\mathrm{T}$ cells), which may suppress optimal IL-21 production from $\mathrm{T}_{\mathrm{FH}}$ cells. IL-2, one of the cytokines regulated by PD-1 ${ }^{64}$, is known to inhibit $\mathrm{T}_{\mathrm{FH}}$ cell generation and function (through STAT5-mediated regulation of Blimp1 expression) ${ }^{65,66}$.

The recent finding that $\mathrm{PD}-1$ might regulate $\mathrm{T}_{\mathrm{FR}}$ cells (i.e. $\mathrm{PD}-1-$ deficiency may enhance the $\mathrm{T}_{\mathrm{FR}}$ number and suppressive functions) further adds to the complex role of PD-1 in regulation of GC reaction ${ }^{67}$. Regardless of the regulatory mechanisms -directly, through $\mathrm{T}_{\mathrm{FH}}$ or indirectly, through $\mathrm{T}_{\mathrm{FR}}$ cells, the lack of interactions between PD-1 and PD-Ls mitigates the GC responses and leads to impaired immunological memory, defective selection of plasma cells and imbalance of bacterial communities in the gut. Microbial dysbiosis in $P d c d 1^{-/}$mice impairs the gut barrier function and leads to a generalized activation of the immune system, which drives the expansion of 
self-reactive $\mathrm{B}$ and $\mathrm{T}$ cells and the production of auto-antibodies ${ }^{18,30}$. These findings emphasize the critical role of PD-1 in the regulation of not only $\mathrm{T}$ cell but also $\mathrm{B}$ cell responses. Under homeostatic conditions, the rheostat function of PD-1 is critical for maintaining the balance of bacterial communities in gut, while during infection is essential for the generation of immunological memory (Fig. 2). By influencing the composition of the gut microbiota, PD-1 is likely to contribute to fine-tuning other major physiological processes in the body, such as the endocrine, cardiovascular or nervous system functions.

\section{Regulation of viral infection by PD-1}

PD-1 plays unique regulatory roles in the control of viral infections. PD-1 clearly attenuates the magnitude of primary responses during acute infection. In an adenovirus-induced hepatitis model, PD-1-deficiency augmented proliferation and accumulation of effector $T$ cells in the liver, and caused rapid clearance of the virus ${ }^{68}$ (Fig. 2). Yet, despite the early virus clearance, $P d c d 1^{-/-}$mice showed severe hepatitis, in contrast with wild-type mice which had prolonged hepatitis with slow viral clearance. These observations indicate that PD-1 may be important to avoid excessive tissue damage during the acute phase of infection. Consistent with this idea, infection of Pdcdl $\lg 1^{-/}$- mice with LCMV clone 13 known to cause viral persistence resulted in death of infected mice because of severe damage to the liver ${ }^{69}$. 
Although transient PD-1 expression may inhibit excessive immune response in acute infection, high and persistent expression of PD-1 causes chronic immune responses. Interestingly, extremely high expression of PD-1 $(\sim 2-3$ logs higher than transiently activated $\mathrm{T}$ cells) was observed on $\mathrm{CD}^{+} \mathrm{T}$ cells in chronic infection with LCMV clone $13^{69}$. Virus-specific PD- $1{ }^{\text {hi }} \mathrm{CD}^{+} \mathrm{T}$ cells were shown to have fallen into a state of anergy or unresponsiveness and therefore were called "exhausted T cells" (to emphasize their lost ability to produce TNF, IFN- $\gamma$ and IL-2) ${ }^{69}$. The PD-1 ${ }^{\text {high }}$ "exhausted" population is not functionally incompetent, but contains memory cells capable of re-expansion and viral clearance upon secondary infection with non-persisting (acute) clone of LCMV armstrong. Interestingly, re-expanded “exhausted" cells retained high PD-1 expression and weak cytokine profile, suggesting the "exhaustion" establishes as a consequence of PD-1 mediated adaptation to pathogens without self damage, resulting in chronic infection ${ }^{70}$. Importantly, transient blockade of the PD-1-PD-L1 pathway by anti-PD-L1 mAb restored the function of "exhausted T cells" and enhanced T cell responses for clearing the viruses. The reversal of exhausted state by PD-L1 blockade can be accomplished even in CD4-depleted "helpless" mice, suggesting that the recovery of cytotoxic properties is CD8 ${ }^{+} \mathrm{T}$ cellintrinsic ${ }^{69}$. A similar high expression of PD-1 was observed in $\mathrm{CD}^{+} \mathrm{T}$ cells from tumor-bearing and aged mice ${ }^{71}$. Although these senescent PD- ${ }^{\text {hi }}$ CD4 ${ }^{+} \mathrm{T}$ cells hardly proliferate in response to TCR stimulation, they still produce osteopontin (a pro-inflammatory cytokine), and have high expression of C/EBP $\alpha$ (normally expressed on myeloid-lineage cells) and diminished expression of c-Myc and cyclin D1. 
The high expression of PD-1 in the chronic phases of immune reactions may be achieved by the marked and irreversible $\mathrm{CpG}$ demethylation in the PD-1 promoter region located $500 \sim 1500 \mathrm{bp}$ upstream of the initiation codon ${ }^{72}$. In addition, this region contains two transcription factor binding sites (NFAT (-1160) ${ }^{73,74}$ and ISRE $(-1040)){ }^{73}$ which are activated by TCR and IFN-dependent pathways, respectively. It is likely that continuous stimulation through antigen receptors (TCR and BCR), in collaboration with inflammatory cytokines (i.e IFNs) causes the demethylation (opening) of the locus, resulting in high expression of PD-1.

As PD-1 blockade after establishment of chronic infection refuels the immune response, PD-1 seems an attractive therapeutic target against chronic infection. However, the outcome of the PD-1 blockade varies depending on the experimental system and the timing of blockade. Further analyses are expected to provide better rationale for the safe and effective application of PD-1 antagonist for the treatment of chronic infection as well as improvement of immune responses after vaccination, or for the use of PD-1 agonist for treatment of fulminant diseases.

\section{PD-1 regulation of tumor growth and metastasis}

The PD-1-PD-L1 pathway plays a pivotal role in dampening immune-surveillance for tumors. The first evidence was provided by the observation that overexpression of PD-L1 on mouse plasmacytoma cell line inhibited the cytolytic activity of $\mathrm{CD}^{+} \mathrm{T}$ cells through engagement with PD-1, which enhanced their growth and invasiveness ${ }^{15}$. In addition, this report clearly demonstrated that $\mathrm{PD}-\mathrm{L} 1^{+}$myeloma 
cells could not produce tumors in $P d c d 1^{-/-}$mice and that the blockade of interaction between PD-L1 and PD-1 activates CD ${ }^{+}$T cells that can attack tumors (Fig. 2). On the other hand, expression of PD-L1 on tumors was reported to provide resistance to T cells by promoting T cell apoptosis through non-PD-1 receptors ${ }^{75}$.

To date, blocking of PD-1-PD-L1 interaction using various systems including antibody blockade of PD-1 and PD-L1, genetic manipulation of PD-1 and DNA vaccination of the extracellular region of PD-1, has been shown to accelerate tumor eradication ${ }^{15,76-79}$. Accumulated data from clinical samples show that high expression of PD-1 ligands on tumors correlates with poor prognosis, which suggested that tumor could escape anti-tumor immunity through ligand engagement of PD-1 on T cells $\left({ }^{80}\right.$ and summarized in ${ }^{81}$ ).

Based on these results, a fully humanized mAb against PD-1 (Nivolumab/ONO4538/MDX-1106/BMS 936558) was developed by immunizing genetically modified mice carrying human Ig loci with human PD-1 molecule ${ }^{82}$. The IgG4 isotype of Nivolumab minimizes complement activation or ADCC, preventing unnecessary cellular toxicity and inflammation. The phase I clinical trial study of Nivolumab was initiated in $2006^{82}$. The cumulative response rates were $18 \%$ (14 of 76 patients) in non-small cell lung cancer (NSCLC), 28\% (26 out of 94 patients) in melanoma and $27 \%$ (9 out of 33 patients) in renal cell carcinoma ${ }^{83}$. Grade $3-4$ drug related adverse events occurred in 14 patients. A clinical trial using an anti-PD-L1 mAb (BMS-936559/MDX-1105) showed similar anti-cancer activity ${ }^{84}$. In these studies, anti-PD-1 and anti-PD-L1 antibody showed the highest rate of antitumor activity of the 
many immunotherapy approaches tested in the clinic for the treatment of cancer during the past 30 years ${ }^{85}$. Accumulating data suggest that the therapeutic use of anti-PD-1 $\mathrm{mAb}$ alone or in combination with other drugs will provide promising therapy for many types of advanced cancer. Nivolumab was recently designated as an orphan drug for malignant melanoma (http://www.accessdata.fda.gov/scripts/opdlisting/oopd/OOPD_Results_2.cfm?Index_N umber $=387612$ ).

\section{Concluding remarks}

A key point in elucidating the function of PD-1 as a rheostat of the immune response was the characterization of the autoimmune phenotype of PD-1-deficient animals, with manifestations that were unique and strikingly different among different genetic backgrounds ${ }^{2-7}$. The background- and organ-specific autoimmune manifestations together with the relatively mild symptoms and late disease onset indicated that PD-1 might regulate the immune response in an antigen-specific fashion. This assumption was supported by further molecular studies revealing the role of PD-1 in inhibiting signal transduction upon specific engagement of antigen receptors. The elucidation of molecular mechanisms of PD-1 functions, together with the identification of its ligands ${ }^{8}$, ${ }^{9}$, allowed for testing of PD-1 involvement in various aspects of the immune response. Another critical observation was that blockade of PD-1- PD-L1 interactions by antibody or genetic manipulation can potentiate $\mathrm{CD}^{+} \mathrm{T}$ cells attacking tumors and viruses ${ }^{15,16} 68$. This finding eventually led to clinical studies investigating the association between 
ligand expression on tumors and prognosis of tumor patients (summarized in ${ }^{81}$ ). The most recent breakthrough was the finding that PD-1 blocking by antibodies is so far the most promising immunotherapy for cancers. This development, in turn, indicates that the immune system effectively recognizes tumor cells, and suggests that rekindling of the immune surveillance, which is likely anergized by a large excess of tumor antigens and chronic stimulation ${ }^{86}$ may be an effective strategy to control malignancies. The success of PD-1 blocking in cancer treatments appears to depend on the unique features of PD-1 briefly discussed in this review, which clearly distinguish PD-1 from other molecules with immune suppressor functions.

Nevertheless, it is important to realize that there are at least $70 \%$ of cancer patients who did not respond well to anti-PD-1 treatment ${ }^{83}$. The exact reason for this unresponsiveness is a target for further investigations. Genetic polymorphisms and cancer cell mutations should be thoroughly investigated to identify clinical markers that could distinguish responders from non-responders. It is also important to test various combination therapies for non-responders. The combination may include stimulation with cancer peptides and combined therapies with blocking agents of other negative regulators, expecting either additive or synergistic effects of anti-tumor activities. These complementary treatments include anti-PD-1 mAb with cytokines (i.e. IFN- $\alpha^{73}$ ), antigen stimulation ${ }^{87}$ and blockade of other immune-inhibitory pathways such as Tim- $3^{88}$, LAG- $3^{89}$ and CTLA- $4^{90}$. A combinational therapy of PD-1 and CTLA-4 monoclonal antibodies resulted in more than $50 \%$ objective response rate for patients with advanced melanoma ${ }^{89}$. Overall, the serendipity journey of PD-1 from bench to 
bedside, once again teaches us that the basic science research is critical for drug discovery and translational medicine.

\section{Acknowledgements}

We thank all colleagues whose works were mentioned in this manuscript. We sincerely appreciate the scientific expertise from N. Minato and his colleagues at Kyoto University over all these years. This work was supported by Core Research for Evolutional Science and Technology Program of the Japan Science and Technology Agency (to T. O.), Grants-in-Aid for Scientific Research from the Japanese Ministry of Education, Culture, Sports, Science and Technology (\#23790534, \#25460363 to S.C.), the Senri Life Science Foundation (to S.C.), Grants-in-Aid for Scientific Research in Priority Areas and RIKEN's President Discretionary Fund (to S. F.).

\section{Competing financial interest}

The authors declare no competing financial interests.

\section{Figure legends}

Figure 1. PD-1 induces $\mathbf{T}$ cell tolerance. PD-1 inhibits $T$ cell receptor signaling pathway through SHP-2. T cells are activated by signal 1 (antigen stimulation), signal 2 (costimulation), and signal 3 (inflammatory cytokines). In naïve T cells, TCR-mediated calcium influx initiates PD-1 transcription by activating NFATc1. In chronically activated ("exhausted") T cells, IFN- $\alpha$ causes prolonged PD-1 transcription through the 
binding of IRF9 to the PD-1 promoter. When its physiological ligand (PD-L1 and PD-L2) binds, PD-1 suppresses $\mathrm{T}$ cell activation and function through the recruitment of SHP-2, which dephosphorylates and inactivates ZAP70, a major integrator of TCR-mediated signaling.

Figure 2. Biological significance of PD-1 signaling. PD-1 is induced on activated $\mathrm{CD}^{+}$and $\mathrm{CD}^{+} \mathrm{T}$ cells, as well as B cells. Inflammatory stimulation induces PD-L1 expression on many types of non-hematopoietic and hematopoietic cells, and PD-L2 expression on antigen-presenting cells. The engagement of PD-1 with its ligands inhibits proliferation and effector function of $\mathrm{T}$ cells and antibody production of $\mathrm{B}$ cells resulting in prevention of autoimmunity and attenuation of anti-tumor and anti-infectious immunity.

Figure 3. Distinct mechanisms of PD-1 and CTLA4 in immune suppression. (a) PD-1 controls the effector phase of the immunity primarily in a cell-intrinsic manner by inducing unresponsiveness through attenuating antigen-specific signals. Autocrine and/or paracrine regulation by PD-1 is also possible by inhibiting cytokine expression. Only $\mathrm{CD}^{+} \mathrm{T}$ cells are shown here. Although not depicted, $\mathrm{CD} 4^{+} \mathrm{T}$ cells interact with antigen presenting $\mathrm{B}$ cells and regulate the quality and quantity of antibodies. (b) CTLA-4 controls particularly the function of activated $\mathrm{CD}^{+} \mathrm{T}$ cells expressing Foxp3. CTLA-4 dominantly captures CD80 and CD86 on APCs and down-modulates the costimulatory activity of CD28 on effector T cells. TGF- $\beta 1$, an important cytokine 
produced by Foxp $3^{+} \mathrm{T}$ cells supports growth and differentiation of Foxp $3^{+} \mathrm{T}$ cells and suppresses diverse immune responses. 


\section{Comments for references with top 10 significance}

1. Ishida et al.

The original report of the isolation and characterization of mouse PD-1 by subtractive cDNA library between stimulated and control thymoma cell lines. PD-1 was induced on murine thymoma cell lines upon apoptotic stimuli.

2. Nishimura et al.

The first demonstrationthatC57BL/6 $\mathrm{Pdcd} \mathrm{I}^{-/}$mice succumb to SLE-like autoimmune manifestations. The data indicated that PD-1 plays a crucial role in maintaining self-tolerance.

\section{Nishimura et al.}

The first report showing that $P d c d 1^{-/-}$mice on BALB/c background developed lethal dilated cadiomyopathy. Together with Ref. 2, this paper revealed that PD-1 deficiency causes autoimmune reaction to different organs, depending on the genetic background.

8. Freeman et al

This report is the first demonstration of PD-L1 as specific ligand for PD-1.

\section{Okazaki et al.}

This paper demonstrated that tyrosine phosphatase SHP-2 associating with cytoplasmic tail of PD-1 down-modulates signaling from the antigen receptor, thus revealing the molecular mechanism by which PD-1 mediates lymphocyte inhibition.

15. Iwai et al. 
The first report to show the involvement of the PD-1/PD-L1 pathway in tumor escape from immune surveillance and the effectiveness of PD-L1 blockade for tumor therapy. 18. Kawamoto et al.

This paper showed that $P d c d 1^{-/}$mice have altered selection of IgA B cells in germinal center of Peyer's patches and reduced quality of IgA plasma cells. The lack of PD-1 action in gut GCs leads to intestinal dysbiosis, impaired mucosal firewall, and generalized activation of the immune system.

80. Thompson et al.

This paper demonstrated that enhanced expression of PD-L1 in primary renal tumors correlated with poor prognosis of patients, and supported the idea of PD-1 blocker application to cancer treatment (Ref. 15) in human.

83. Topalian et al.

The first comprehensive study on efficacy and safety of human PD-1 antibody in cancer patients.

84. Brahmer et al.

The first comprehensive study on efficacy and safety of human PD-L1 antibody in cancer patients. 


\section{References}

1. Ishida, Y., Agata, Y., Shibahara, K. \& Honjo, T. Induced expression of PD-1, a novel member of the immunoglobulin gene superfamily, upon programmed cell death. Embo J 11, 3887-3895 (1992).

2. Nishimura, H., Nose, M., Hiai, H., Minato, N. \& Honjo, T. Development of lupus-like autoimmune diseases by disruption of the PD-1 gene encoding an ITIM motif-carrying immunoreceptor. Immunity 11, 141-151 (1999).

3. Nishimura, H. et al. Autoimmune dilated cardiomyopathy in PD-1 receptor-deficient mice. Science 291, 319-322 (2001).

4. Wang, J. et al. Establishment of NOD-Pdcd1-/- mice as an efficient animal model of type I diabetes. Proc Natl Acad Sci U S A 102, 11823-11828 (2005).

5. Wang, J. et al. PD-1 deficiency results in the development of fatal myocarditis in MRL mice. Int Immunol 22, 443-452 (2010).

6. Yoshida, T., Jiang, F., Honjo, T. \& Okazaki, T. PD-1 deficiency reveals various tissue-specific autoimmunity by $\mathrm{H}-2 \mathrm{~b}$ and dose-dependent requirement of $\mathrm{H}-2 \mathrm{~g} 7$ for diabetes in NOD mice. Proc Natl Acad Sci U S A 105, 3533-3538 (2008).

7. Okazaki, T. et al. Hydronephrosis associated with antiurothelial and antinuclear autoantibodies in BALB/c-Fcgr2b-/-Pdcd1-/- mice. J Exp Med 202, 1643-1648 (2005).

8. Freeman, G.J. et al. Engagement of the PD-1 immunoinhibitory receptor by a novel B7 family member leads to negative regulation of lymphocyte activation. $J$ Exp Med 192, 1027-1034 (2000).

9. Latchman, Y. et al. PD-L2 is a second ligand for PD-1 and inhibits T cell activation. Nat Immunol 2, 261-268 (2001).

10. Chemnitz, J.M., Parry, R.V., Nichols, K.E., June, C.H. \& Riley, J.L. SHP-1 and SHP-2 associate with immunoreceptor tyrosine-based switch motif of programmed death 1 upon primary human $\mathrm{T}$ cell stimulation, but only receptor ligation prevents T cell activation. J Immunol 173, 945-954 (2004).

11. Okazaki, T., Maeda, A., Nishimura, H., Kurosaki, T. \& Honjo, T. PD-1 immunoreceptor inhibits B cell receptor-mediated signaling by recruiting src homology 2-domain-containing tyrosine phosphatase 2 to phosphotyrosine. Proc 
Natl Acad Sci U S A 98, 13866-13871 (2001).

12. Parry, R.V. et al. CTLA-4 and PD-1 receptors inhibit T-cell activation by distinct mechanisms. Mol Cell Biol 25, 9543-9553 (2005).

13. Yokosuka, T. et al. Programmed cell death 1 forms negative costimulatory microclusters that directly inhibit $\mathrm{T}$ cell receptor signaling by recruiting phosphatase SHP2. J Exp Med 209, 1201-1217 (2012).

14. Sheppard, K.A. et al. PD-1 inhibits T-cell receptor induced phosphorylation of the ZAP70/CD3zeta signalosome and downstream signaling to PKCtheta. FEBS Lett 574, 37-41 (2004).

15. Iwai, Y. et al. Involvement of PD-L1 on tumor cells in the escape from host immune system and tumor immunotherapy by PD-L1 blockade. Proc Natl Acad Sci U S A 99, 12293-12297 (2002).

16. Iwai, Y., Terawaki, S. \& Honjo, T. PD-1 blockade inhibits hematogenous spread of poorly immunogenic tumor cells by enhanced recruitment of effector T cells. Int Immunol 17, 133-144 (2005).

17. Good-Jacobson, K.L. et al. PD-1 regulates germinal center B cell survival and the formation and affinity of long-lived plasma cells. Nat Immunol 11, 535-542 (2010).

18. Kawamoto, S. et al. The inhibitory receptor PD-1 regulates IgA selection and bacterial composition in the gut. Science 336, $485-489$ (2012).

19. Bour-Jordan, H. et al. Intrinsic and extrinsic control of peripheral T-cell tolerance by costimulatory molecules of the CD28/ B7 family. Immunol Rev 241, 180-205 (2011).

20. Walker, L.S. Treg and CTLA-4: Two intertwining pathways to immune tolerance. J Autoimmun 45, 49-57 (2013).

21. Zhang, X. et al. Structural and functional analysis of the costimulatory receptor programmed death-1. Immunity 20, 337-347 (2004).

22. Lazar-Molnar, E. et al. Crystal structure of the complex between programmed death-1 (PD-1) and its ligand PD-L2. Proc Natl Acad Sci U S A 105, 10483-10488 (2008).

23. Lin, D.Y. et al. The PD-1/PD-L1 complex resembles the antigen-binding Fv domains of antibodies and T cell receptors. Proc Natl Acad Sci U S A 105, 3011-3016 (2008). 
24. Shiratori, T. et al. Tyrosine phosphorylation controls internalization of CTLA-4 by regulating its interaction with clathrin-associated adaptor complex AP-2. Immunity 6, 583-589 (1997).

25. Agata, Y. et al. Expression of the PD-1 antigen on the surface of stimulated mouse T and B lymphocytes. Int Immunol 8, 765-772 (1996).

26. Nishimura, H. et al. Developmentally regulated expression of the PD-1 protein on the surface of double-negative (CD4-CD8-) thymocytes. Int Immunol 8, 773-780 (1996).

27. Yamazaki, T. et al. Expression of programmed death 1 ligands by murine T cells and APC. J Immunol 169, 5538-5545 (2002).

28. Keir, M.E. et al. Tissue expression of PD-L1 mediates peripheral T cell tolerance. J Exp Med 203, 883-895 (2006).

29. Moll, M. et al. Severe functional impairment and elevated PD-1 expression in CD1d-restricted NKT cells retained during chronic HIV-1 infection. Eur $J$ Immunol 39, 902-911 (2009).

30. Okazaki, T. et al. PD-1 and LAG-3 inhibitory co-receptors act synergistically to prevent autoimmunity in mice. $J$ Exp Med 208, 395-407 (2011).

31. Liang, S.C. et al. Regulation of PD-1, PD-L1, and PD-L2 expression during normal and autoimmune responses. Eur J Immunol 33, 2706-2716 (2003).

32. Keir, M.E., Butte, M.J., Freeman, G.J. \& Sharpe, A.H. PD-1 and its ligands in tolerance and immunity. Annu Rev Immunol 26, 677-704 (2008).

33. Okazaki, T. et al. Autoantibodies against cardiac troponin I are responsible for dilated cardiomyopathy in PD-1-deficient mice. Nat Med 9, 1477-1483 (2003).

34. Felix, S.B. \& Staudt, A. Non-specific immunoadsorption in patients with dilated cardiomyopathy: mechanisms and clinical effects. Int $J$ Cardiol 112, 30-33 (2006).

35. Prokunina, L. et al. A regulatory polymorphism in PDCD1 is associated with susceptibility to systemic lupus erythematosus in humans. Nat Genet 32, 666-669 (2002).

36. Nielsen, C., Hansen, D., Husby, S., Jacobsen, B.B. \& Lillevang, S.T. Association of a putative regulatory polymorphism in the PD-1 gene with susceptibility to type 1 diabetes. Tissue Antigens 62, 492-497 (2003).

37. James, E.S. et al. PDCD1: a tissue-specific susceptibility locus for inherited 
inflammatory disorders. Genes Immun 6, 430-437 (2005).

38. Rui, Y., Honjo, T. \& Chikuma, S. Programmed cell death 1 inhibits inflammatory helper T-cell development through controlling the innate immune response. Proc Natl Acad Sci U S A 110, 16073-16078 (2013).

39. Huard, B. et al. Characterization of the major histocompatibility complex class II binding site on LAG-3 protein. Proc Natl Acad Sci U S A 94, 5744-5749 (1997).

40. Workman, C.J., Dugger, K.J. \& Vignali, D.A. Cutting edge: molecular analysis of the negative regulatory function of lymphocyte activation gene-3. J Immunol 169, 5392-5395 (2002).

41. Fontenot, J.D., Gavin, M.A. \& Rudensky, A.Y. Foxp3 programs the development and function of CD4+CD25+ regulatory T cells. Nat Immunol 4, 330-336 (2003).

42. Kulkarni, A.B. et al. Transforming growth factor beta 1 null mutation in mice causes excessive inflammatory response and early death. Proc Natl Acad Sci US A 90, 770-774 (1993).

43. Shull, M.M. et al. Targeted disruption of the mouse transforming growth factor-beta 1 gene results in multifocal inflammatory disease. Nature 359, 693-699 (1992).

44. Bachmann, M.F., Kohler, G., Ecabert, B., Mak, T.W. \& Kopf, M. Cutting edge: lymphoproliferative disease in the absence of CTLA-4 is not T cell autonomous. J Immunol 163, 1128-1131 (1999).

45. Smyk-Pearson, S.K., Bakke, A.C., Held, P.K. \& Wildin, R.S. Rescue of the autoimmune scurfy mouse by partial bone marrow transplantation or by injection with T-enriched splenocytes. Clin Exp Immunol 133, 193-199 (2003).

46. Li, M.O. \& Flavell, R.A. TGF-beta: a master of all T cell trades. Cell 134, 392-404 (2008).

47. Huber, S. \& Schramm, C. TGF-beta and CD4+CD25+ regulatory T cells. Front Biosci 11, 1014-1023 (2006).

48. Miyao, T. et al. Plasticity of Foxp3(+) T cells reflects promiscuous Foxp3 expression in conventional $\mathrm{T}$ cells but not reprogramming of regulatory $\mathrm{T}$ cells. Immunity 36, 262-275 (2012).

49. Stamper, C.C. et al. Crystal structure of the B7-1/CTLA-4 complex that inhibits 
human immune responses. Nature 410, 608-611 (2001).

50. Schwartz, J.C., Zhang, X., Fedorov, A.A., Nathenson, S.G. \& Almo, S.C. Structural basis for co-stimulation by the human CTLA-4/B7-2 complex. Nature 410, 604-608 (2001).

51. Qureshi, O.S. et al. Trans-endocytosis of CD80 and CD86: a molecular basis for the cell-extrinsic function of CTLA-4. Science 332, 600-603 (2011).

52. Nishimura, H., Minato, N., Nakano, T. \& Honjo, T. Immunological studies on PD-1 deficient mice: implication of PD-1 as a negative regulator for $\mathrm{B}$ cell responses. Int Immunol 10, 1563-1572 (1998).

53. Fagarasan, S. \& Honjo, T. T-Independent immune response: new aspects of B cell biology. Science 290, 89-92. (2000).

54. Haas, K.M. Programmed cell death 1 suppresses B-1b cell expansion and long-lived IgG production in response to T cell-independent type 2 antigens. $J$ Immunol 187, 5183-5195 (2011).

55. Martin, F. \& Kearney, J.F. B1 cells: similarities and differences with other B cell subsets. Curr Opin Immunol 13, 195-201. (2001).

56. Muramatsu, M. et al. Class switch recombination and hypermutation require activation-induced cytidine deaminase (AID), a potential RNA editing enzyme. Cell 102, 553-563 (2000).

57. Haynes, N.M. et al. Role of CXCR5 and CCR7 in follicular Th cell positioning and appearance of a programmed cell death gene-1high germinal center-associated subpopulation. J Immunol 179, 5099-5108 (2007).

58. Linterman, M.A. et al. Foxp3+ follicular regulatory $\mathrm{T}$ cells control the germinal center response. Nat Med 17, 975-982 (2011).

59. Chung, Y. et al. Follicular regulatory $\mathrm{T}$ cells expressing Foxp3 and Bcl-6 suppress germinal center reactions. Nat Med 17, 983-988 (2011).

60. Wollenberg, I. et al. Regulation of the germinal center reaction by Foxp3+ follicular regulatory T cells. J Immunol 187, 4553-4560 (2011).

61. Linterman, M.A. et al. IL-21 acts directly on B cells to regulate Bcl-6 expression and germinal center responses. J Exp Med 207, 353-363 (2010).

62. Zotos, D. et al. IL-21 regulates germinal center B cell differentiation and proliferation through a B cell-intrinsic mechanism. $J$ Exp Med 207, 365-378 (2010). 
63. Kuchen, S. et al. Essential role of IL-21 in B cell activation, expansion, and plasma cell generation during CD4+ T cell-B cell collaboration. J Immunol 179, 5886-5896 (2007).

64. Chikuma, S. et al. PD-1-mediated suppression of IL-2 production induces CD8+ T cell anergy in vivo. J Immunol 182, 6682-6689 (2009).

65. Nurieva, R.I. et al. STAT5 protein negatively regulates T follicular helper (Tfh) cell generation and function. J Biol Chem 287, 11234-11239 (2012).

66. Johnston, R.J., Choi, Y.S., Diamond, J.A., Yang, J.A. \& Crotty, S. STAT5 is a potent negative regulator of TFH cell differentiation. $J$ Exp Med 209, 243-250 (2012).

67. Sage, P.T., Francisco, L.M., Carman, C.V. \& Sharpe, A.H. The receptor PD-1 controls follicular regulatory $\mathrm{T}$ cells in the lymph nodes and blood. Nat Immunol 14, 152-161 (2013).

68. Iwai, Y., Terawaki, S., Ikegawa, M., Okazaki, T. \& Honjo, T. PD-1 inhibits antiviral immunity at the effector phase in the liver. $J$ Exp Med 198, 39-50 (2003).

69. Barber, D.L. et al. Restoring function in exhausted CD8 T cells during chronic viral infection. Nature 439, 682-687 (2006).

70. Utzschneider, D.T. et al. T cells maintain an exhausted phenotype after antigen withdrawal and population reexpansion. Nat Immunol 14, 603-610 (2013).

71. Shimatani, K., Nakashima, Y., Hattori, M., Hamazaki, Y. \& Minato, N. PD-1+ memory phenotype CD4+ $\mathrm{T}$ cells expressing C/EBPalpha underlie $\mathrm{T}$ cell immunodepression in senescence and leukemia. Proc Natl Acad Sci U S A 106, 15807-15812 (2009).

72. Youngblood, B. et al. Chronic virus infection enforces demethylation of the locus that encodes PD-1 in antigen-specific CD8(+) T cells. Immunity 35, 400-412 (2011).

73. Terawaki, S. et al. IFN-alpha directly promotes programmed cell death-1 transcription and limits the duration of T cell-mediated immunity. J Immunol 186, 2772-2779 (2011).

74. Oestreich, K.J., Yoon, H., Ahmed, R. \& Boss, J.M. NFATc1 regulates PD-1 expression upon T cell activation. J Immunol 181, $4832-4839$ (2008).

75. Dong, H. et al. Tumor-associated B7-H1 promotes T-cell apoptosis: a potential 
mechanism of immune evasion. Nat Med 8, 793-800 (2002).

76. Curiel, T.J. et al. Blockade of B7-H1 improves myeloid dendritic cell-mediated antitumor immunity. Nat Med 9, 562-567 (2003).

77. Strome, S.E. et al. B7-H1 blockade augments adoptive T-cell immunotherapy for squamous cell carcinoma. Cancer Res 63, 6501-6505 (2003).

78. Blank, C. et al. PD-L1/B7H-1 inhibits the effector phase of tumor rejection by $\mathrm{T}$ cell receptor (TCR) transgenic CD8+ T cells. Cancer Res 64, 1140-1145 (2004).

79. Hirano, F. et al. Blockade of B7-H1 and PD-1 by monoclonal antibodies potentiates cancer therapeutic immunity. Cancer Res 65, 1089-1096 (2005).

80. Thompson, R.H. et al. Costimulatory B7-H1 in renal cell carcinoma patients: Indicator of tumor aggressiveness and potential therapeutic target. Proc Natl Acad Sci U S A 101, 17174-17179 (2004).

81. Okazaki, T. \& Honjo, T. PD-1 and PD-1 ligands: from discovery to clinical application. Int Immunol 19, 813-824 (2007).

82. Brahmer, J.R. et al. Phase I study of single-agent anti-programmed death-1 (MDX-1106) in refractory solid tumors: safety, clinical activity, pharmacodynamics, and immunologic correlates. J Clin Oncol 28, 3167-3175 (2010).

83. Topalian, S.L. et al. Safety, activity, and immune correlates of anti-PD-1 antibody in cancer. $N$ Engl J Med 366, 2443-2454 (2012).

84. Brahmer, J.R. et al. Safety and activity of anti-PD-L1 antibody in patients with advanced cancer. $N$ Engl J Med 366, 2455-2465 (2012).

85. Ribas, A. Tumor immunotherapy directed at PD-1. $N$ Engl J Med 366, 2517-2519 (2012).

86. Sotomayor, E.M., Borrello, I. \& Levitsky, H.I. Tolerance and cancer: a critical issue in tumor immunology. Crit Rev Oncog 7, 433-456 (1996).

87. Song, M.Y., Park, S.H., Nam, H.J., Choi, D.H. \& Sung, Y.C. Enhancement of vaccine-induced primary and memory CD $8(+)$ T-cell responses by soluble PD-1. J Immunother 34, 297-306 (2011).

88. Sakuishi, K. et al. Targeting Tim-3 and PD-1 pathways to reverse T cell exhaustion and restore anti-tumor immunity. J Exp Med 207, 2187-2194 (2010).

89. Wolchok, J.D. et al. Nivolumab plus Ipilimumab in Advanced Melanoma. $N$ Engl J Med 2013, 2 (2013). 
90. Curran, M.A., Montalvo, W., Yagita, H. \& Allison, J.P. PD-1 and CTLA-4 combination blockade expands infiltrating $\mathrm{T}$ cells and reduces regulatory $\mathrm{T}$ and myeloid cells within B16 melanoma tumors. Proc Natl Acad Sci U S A 107, 4275-4280 (2010). 
Fig. 1

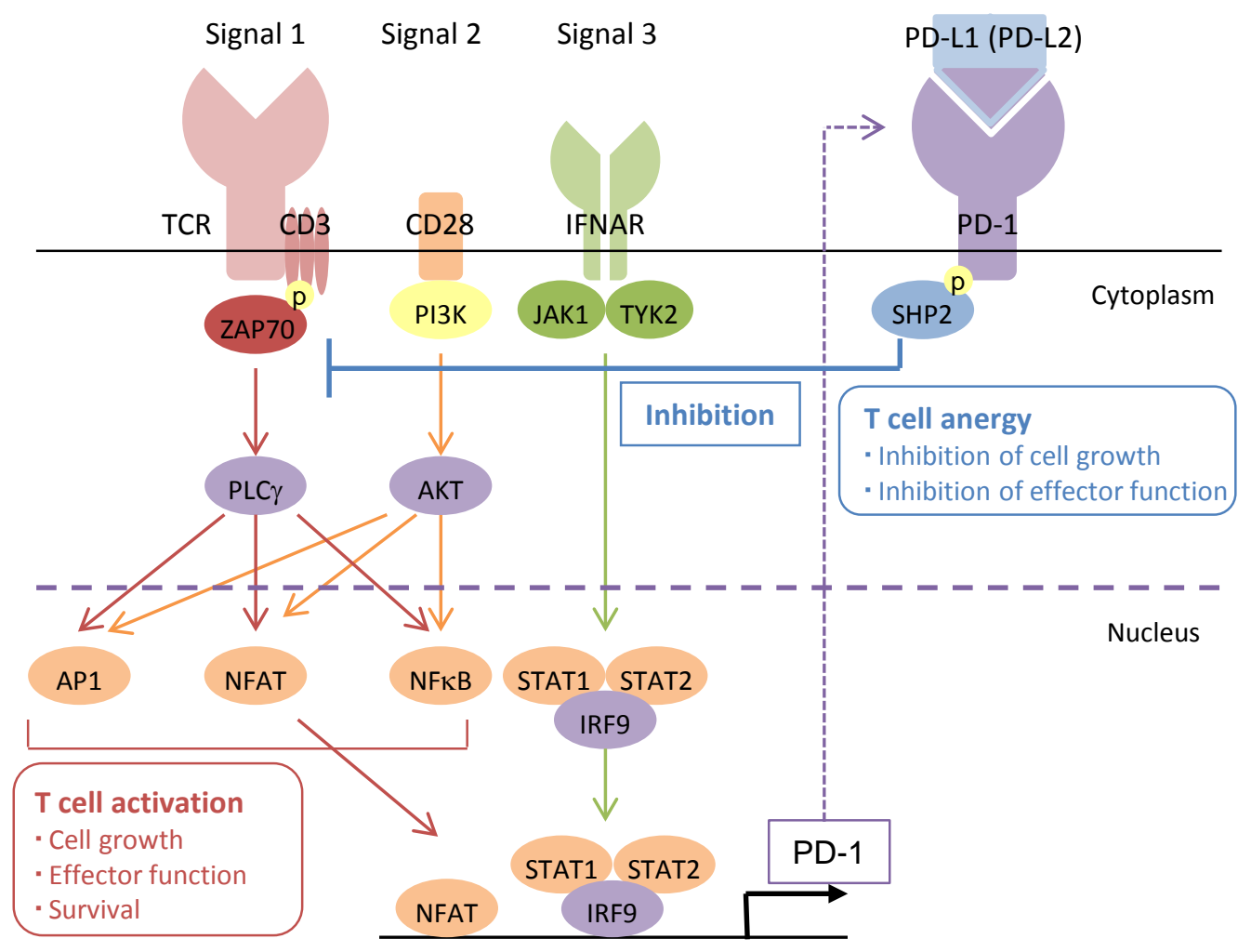




\section{Fig. 2}

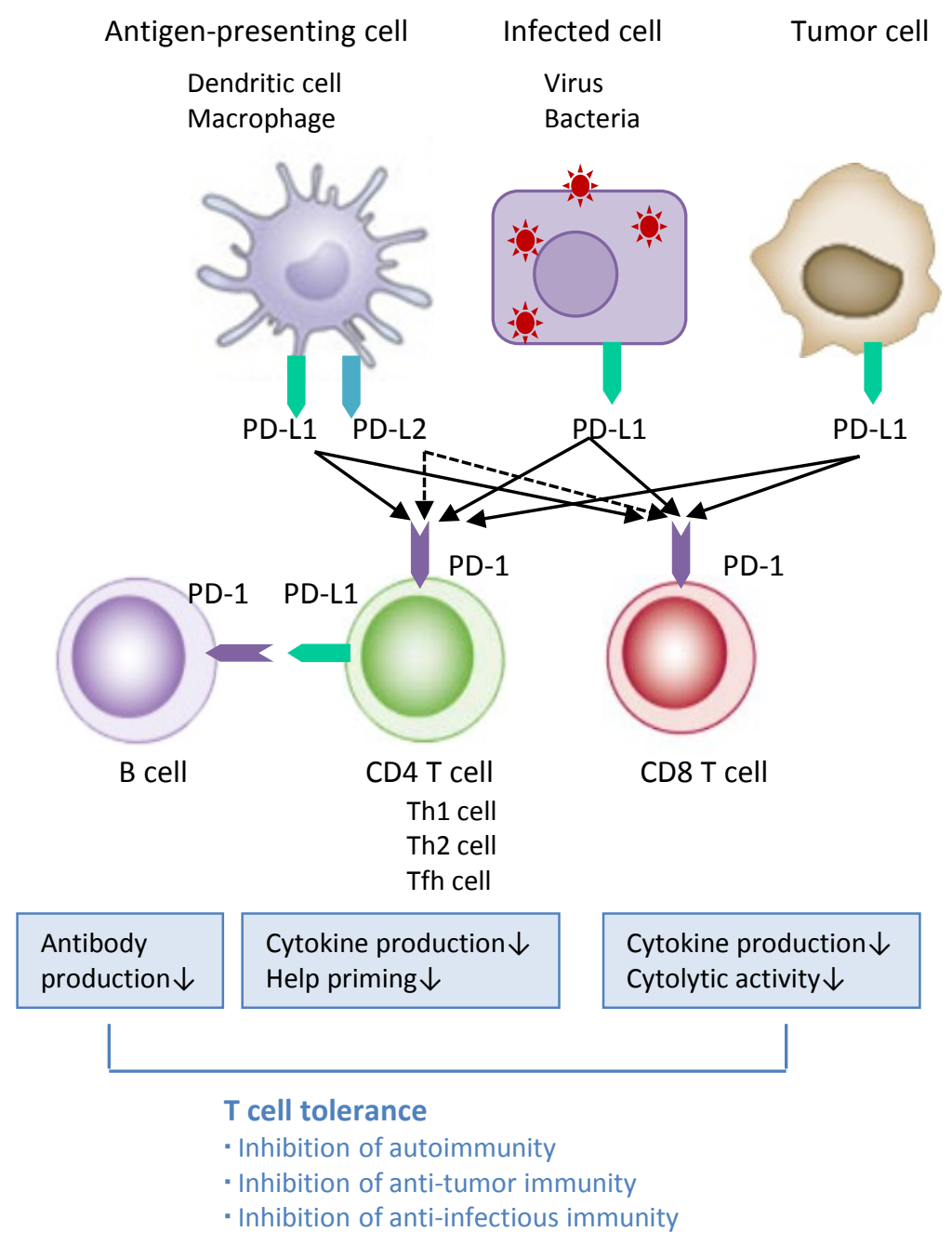




\section{Fig.3}

A

Infected cells/tumor

Effector T cell (CD8)

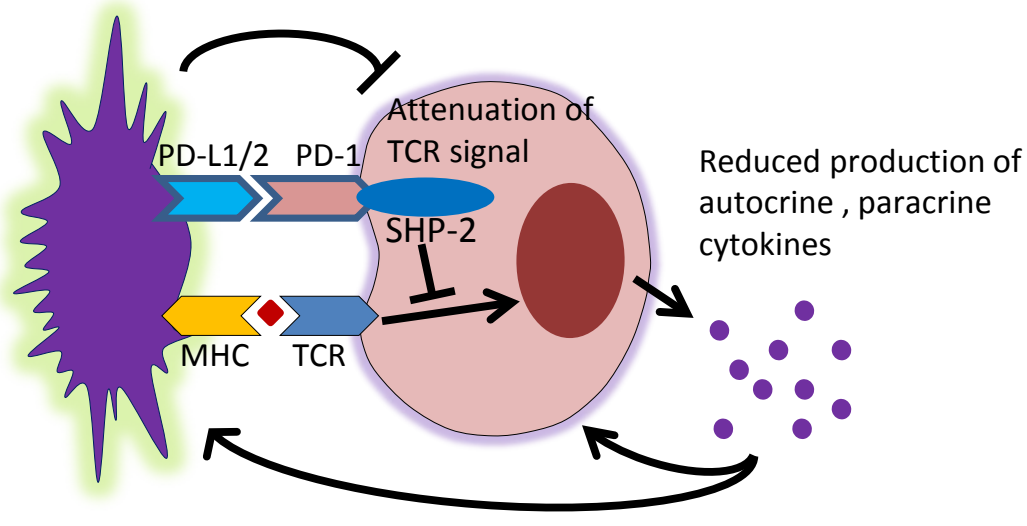

B

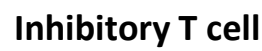

\section{APC for priming}

Effector T cell

Depletion of CD80 and CD86

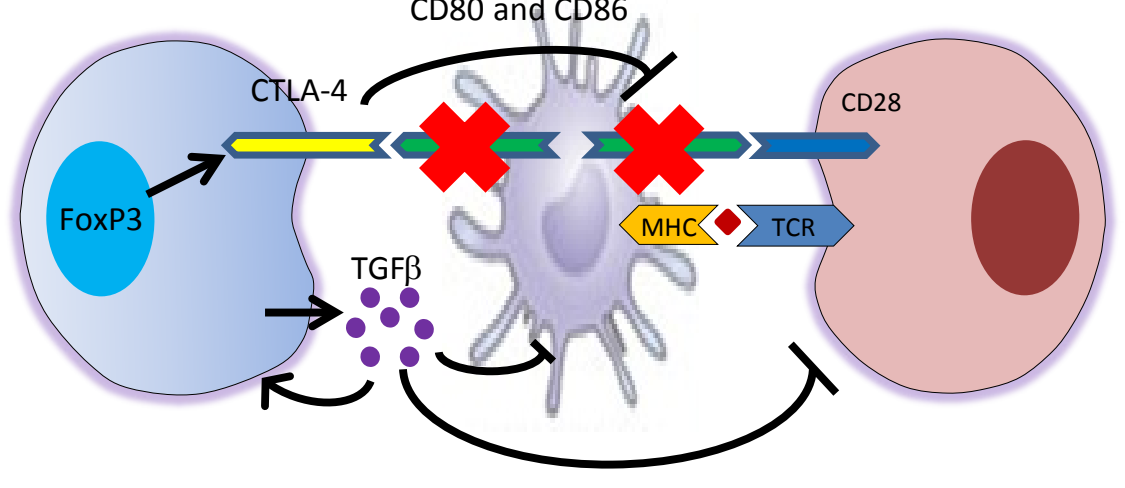

